CDD: 160

\title{
MATRIX-BASED LOGIC FOR AVOIDING PARADOXES AND ITS PARACONSISTENT ALTERNATIVE
}

\author{
PAUL WEINGARTNER
}

Department of Philosophy

University of Salzburg and

Institut für Wissenschaftstheorie

Salzburg, A-5020.

AUSTRIA

paul.weingartner@sbg.ac.at

\begin{abstract}
The present article shows that there are consistent and decidable manyvalued systems of propositional logic which satisfy two or all the three criteria for non-trivial inconsistent theories by da Costa (1974). The weaker one of these paraconsistent system is also able to avoid a series of paradoxes which come up when classical logic is applied to empirical sciences. These paraconsistent systems are based on a 6-valued system of propositional logic for avoiding difficulties in several domains of empirical science (Weingartner (2009)).
\end{abstract}

Keywords: Paraconsistent system. Paradox. Relevance. Many-valued logic.

\section{INTRODUCTION}

Newton da Costa is wellknown for his contributions to Paraconsistent Logics, more correctly it can be said he is the founder of this relatively new domain of Logic. I remember that I saw Newton for the first time at the international congress for Logic, Methodology and Philosophy of Science 1987 in Moscow. I was impressed by his invited lecture "Logic and Pragmatic Truth". Later we met several times on congresses in Europe and Southamerica.

I dedicate the following article to Newton da Costa from whom I got many important stimulations for my thought.

Manuscrito - Rev. Int. Fil., Campinas, v. 34, n. 1, p. 365-388, jan.-jun. 2011. 
Before I begin let me give some general remarks on how I see the approach on Paraconsistent Logic. First of all, since I am a realist, I do not believe that reality can contain contradictions. On the other hand, since man is imperfect, both his thinking and his oral and written communications can contain contradictions. Therefore also our descriptions, hypotheses and theories about reality can contain contradictions. In this sense there is justified motivation to investigate epistemic systems which allow inconsistencies and try to handle them in a logical deductive way. It is not so well known that already Aristotle investigated deductive arguments containing contradictory premises.

In his seminal article "On the theory of inconsistent formal systems" Newton da Costa formulated three principles for calculi which 'are intended to serve as bases for non-trivial inconsistent theories' (da Costa (1974, p. 498)).

DC1 "In these calculi the principle of contradiction, $\neg(A \wedge \neg A)$, must not be a valid schema";

DC2 "From two contradictory formulas $A$ and non- $A$ it will not in general be possible to deduce an arbitrary formula $B$ ";

DC3 Each calculus "must contain the most part of the schemata and rules of CL (Classical Logic) which do not interfere with the first two conditions".

A system which satisfies DC2 and DC3 will be called a weak paraconsistent system. One which satisfies DC1, DC2 and DC3 will be called a strong paraconsistent system.

The purpose of this paper is to construct two models in the sense of matrix calculi of propositional logic which satisfy either DC2 and DC3 or DC1, DC2 and DC3. These models are (weak/strong) paraconsistent alternatives of a basic logic (called RMQ) for the application in empirical sciences and especially also in modern physics (Weingart- 
ner (2009)). This basic logic is a finite matrix system and hence it contains its own semantics. It has the following properties:

1) RMQ is a 6-valued matrix system (3 values for truth, 3 for falsity) and so it contains its own semantics. Every well formed formula of RMQ is unambiguously determined by a particular matrix which contains $6^{n}$ values for $n(n=1,2, \ldots)$ different propositional variables.

2) RMQ is motivated by two criteria called replacement (RC) and reduction (RD) which avoid difficulties in the application of logic (see below 6 and 7 ).

3) RMQ is consistent and decidable.

4) RMQ has the finite model property.

5) RMQ has two concepts of validity: a weaker one (classically valid which is identical with materially valid) and a stronger one (strictly valid). All theorems of two-valued Classical Logic (Classical Propositional Calculus CPC) are at least classically valid, that is materially valid, in RMQ. Only a restricted class of them are strictly valid in RMQ. Therefore: RMQ satisfies DC3 above.

6) The validity of a proposition is decided by calculating the highest value $(c v)$ in its matrix. If $c v=3$ the proposition (formula) is classically valid, that is materially valid. If $c v=2$ the proposition (formula) is strictly valid.

7) The strictly valid theorems of RMQ avoid a great number of wellknown paradoxes in the domain of scientific explanation, law statements, disposition predicates, verisimilitude, theory of human actions, deontic logic ... etc. ${ }^{1}$

\footnotetext{
${ }^{1}$ See below 2.1 and theorems in 3.2 and 3.3 .
} 
8) The strictly valid theorems of RMQ avoid the well known difficulties when logic is applied to physics; especially those with commensurability, distributivity and with Bell's inequalities. See Weingartner (2009, Section 4.3, p. 148 - 153).

9) RMQ is closed under transitivity of implication, under modus ponens, and under equivalence substitution.

10) RMQ contains a modal system with 14 modalities which is close to the modal system $\mathrm{T}$ (of Feys) concerning the theorems with one modal operator (no iteration) applied to well formed formulas.

The paper will be divided into the following sections: In section $\mathbf{2}$ the two criteria RC and RD will be described. The system RMQ will be defined in section 3 and 3.1. In section 3.2 those theorems of RMQ will be statet, which are only classically or materially valid, in $\mathbf{3 . 3}$ those which are strictly valid in RMQ and satisfy RC and RD. In section 4 the two paraconsistent alternatives to RMQ are presented. The first (weak paraconsistent system) is obtained from RMQ by weakening the negation of RMQ. The second is obtained from RMQ by weakening its conjunction. Advantages and disadvantages of these two systems are discussed. The modal logic included of RMQ will not be discussed in this paper.See Weingartner (2009).

\section{THE MOTIVATING CRITERIA RC AND RD}

The criteria RC and RD have been introduced in order to avoid paradoxes when logic is applied to empirical sciences. Such paradoxes have been described in several papers (Weingartner, Schurz (1986), Weingartner (2001)).. For difficulties in the domain of Quantum Physics see Mittelstaedt $(1978,2004)$, Weingartner $(2004,2009,2010)$. The criteria originate in a paper devoted to give a solution of the problem of verisimilitude in the sense of rehabilitating Popper's original idea 
by restricting the logical consequence class to relevant consequentelements only (cf. Schurz, Weingartner (1987). Further developments are in Schurz (1991) and Weingartner (2000) and Schurz, Weingartner (2009)).

\subsection{The Replacement Criterion (RC)}

$\mathrm{RC} \alpha$ is a relevant (nonredundant) consequence of $A$ iff $A \vdash \alpha$ or $A \Rightarrow$ $\alpha$ (according to $\mathrm{CPC}$ ) and it is not the case that a propositional variable is replaceable in $\alpha$ on some of its occurrences by any other propositional variable salva validate of $A \vdash \alpha$ or $A \Rightarrow \alpha$.

Or in other words:

A classically valid argument $A \Rightarrow \alpha$ is replacement invariant (replacement restricted) iff it is the case (it is not the case) that some propositional variable is replaceable in $\alpha$ on some of its occurrences by any other propositional variable salva validitate of $A \Rightarrow \alpha .^{2}$

In order to avoid misunderstanding we might answer the following questions: (1) What does the expression "by any other propositional variable" mean: it means an arbitrary other variable which is a wff of the system of propositional logic. Since any propositional variable is an atomic wff we could also say: by an arbitrary wff. But RC could also be formulated by using just 'wff' instead of 'atomic wff' (or propositional variable). This has been proved in Schurz, Weingartner (1987).

(2) What does it mean "on some of its occurrences"? This presupposes a necessary distinction between "propositional variable (wff)"

\footnotetext{
${ }^{2}$ The criteria RC and RD can also be formulated more generally for FirstOrder Predicate Logic with Identity. In this case a clause for identity formulas has to be added in RC (cf. the references in note above). Since RMQ deals only with Propositional Logic we need only the more specific formulation.
} 
and "particular occurrence of a propositional variable (wff)": Thus in $p \Rightarrow(p \vee q)$ the variable ' $q$ ' has one occurrence whereas in $p \Rightarrow$ $((p \vee q) \wedge(q \vee \neg q))$ it has three occurrences. And the variable ' $q$ ' can be replaced salva validitate by an arbitrary other variable in both valid formulas either on one of its occurrences or on all the three of its occurrences (uniformly, i.e., on all three occurrences by the same variable).

It is worth mentioning that already Aristotle's syllogistics contains a relevance criterion which is weaker than the replacement criterion (applied to predicates), but which is contained in it: Every correct syllogistic inference obeys the principle: there are no (new) predicates in the conclusion (consequence) which are not contained in the premises.

$\mathrm{RC}$ rules out the following theorems which give rise to difficulties and paradoxes.

(1) Ex falso quodlibet:

$\neg p \Rightarrow(p \rightarrow q)$; where ' $\Rightarrow$ ' stands for ' $\vdash\left(\ldots \rightarrow \_\right.$)'.

Culprit for: Carnap's Paradox of Disposition Predicates, Paradox of Derived Obligation, Paradox of Commitment.

(2) Principles of addition:

$p \Rightarrow(p \vee q)$.

Culprit for: Hesse's Confirmation Paradox, Goodman's Paradox, Ross's Paradox, Paradox of Free Choice, Paradox of Versimilitude, Paradox of Irrelevant Explanandum Components. ${ }^{3}$

(3) Adding premise:

$p \Rightarrow(q \rightarrow p)$

Culprit for: Assumption of Commensurability (cf. below), Paradox of Total Theoretical Explanation, Paradox of Irrelevant Law Specification.

\footnotetext{
${ }^{3}$ Since most of the well-known systems of Relevant Logic (E,R,... etc.) all contain the principle of addition as valid, they are unable to avoid these and other paradoxes.
} 
(4) Assumption of commensurability: $p \Leftrightarrow[(p \wedge q) \vee(p \wedge \neg q)]$. The direction $\Rightarrow$ is ruled out.

(5) Logical assumption of CPC underlying Bell's inequalities: $(p \wedge r) \Rightarrow[(p \wedge q) \vee(r \wedge \neg q)]$

It is easily seen that according to the replacement criterion all the five CPC valid principles are irrelevant because ' $q$ ' can be replaced (on one or two occurrences) by any arbitrary variable (standing for any proposition, simple, or complex) salva validitate (veritate).

\subsection{The Reduction Criterion (RD)}

$\mathrm{RD} \alpha$ is a reduced consequence of $A$ iff

(a) $\alpha$ is a relevant (RC-) consequence of $A$.

(b) $\alpha$ is in conjunctive normal form ( $\mathrm{CN}$-form) and not logically equivalent to a conjunction of $\mathrm{CN}$-formulas which are shorter than $\alpha$.

$\mathrm{RD}$ rules out the following theorems which give rise to redundancies, difficulties, and paradoxes. (1) Reduction of redundancies.

(1a) $p \Rightarrow p \wedge p \wedge \ldots$

(1b) $p \Rightarrow p \vee p \vee \ldots$

$\mathrm{RD}$ allows only the opposite arrows $\Leftarrow$.

(2) Reduction to conjuncts as most informative consequence elements. The following directions which lead from conjuncts to disjuncts or from separated parts to conjuncts are ruled out: Only the opposite directions $\Leftarrow$ (not listed here) are permitted by $\mathrm{RD}$.

(2a) $[p \wedge(q \vee \neg q)] \Rightarrow[(p \wedge q) \vee(p \wedge \neg q)]$

(2b) $[p \wedge(q \vee r)] \Rightarrow[(p \wedge q) \vee(p \wedge r)]$ 
(2c) $[(p \vee q) \wedge(p \vee r)] \Rightarrow[p \vee(q \wedge r)]$

$(2 \mathrm{~d})[(r \rightarrow p) \wedge(r \rightarrow q)] \Rightarrow[r \rightarrow(p \wedge q)]$

Although the left parts like $p \wedge(q \vee r),(p \vee q) \wedge(p \vee r)$ are not yet reduced consequences by $\mathrm{RD}$, they are first steps which can be easily turned into reduced consequences by splitting up the conjunctions into their parts.

Or in other words: the right direction of the distribution laws is that direction which leads to a consequent (conclusion) which is a conjunction of reduced consequences.

Consequently distribution laws, which are problematic when logic is applied to Quantum Physics, are ruled out, whereas the acceptable ones (leading to conjuncts) are permitted; they hold necessarily in RMQ.

\section{THE SYSTEM RMQ}

It was one of the purposes when constructing RMQ, to find a semantics with the help of finite matrices (truth tables), which approximates the effects of the relevance restriction of the replacement (RC) and reduction $(\mathrm{RD})$ criteria in such a way that what is classically valid but redundant or irrelevant or leading to disjuncts by those criteria, is materially valid but strictly invalid in RMQ. This was indeed achieved by RMQ as one can see from the theorems listed in Sections $\mathbf{3 . 2}$ and 3.3 below.

\subsection{Definition of the system RMQ}

The system RMQ can be defined as the set of all formulas (wffs) which are satisfied by the matrix $M=\langle T, F, \neg, \vee, \wedge, \rightarrow, L\rangle$, where $T=$ $\{1,2,3\}, F=\{4,5,6\}$, and the operations $\neg, \vee, \wedge, \rightarrow, L$ are defined as follows: ${ }^{4}$

\footnotetext{
${ }^{4}$ Where 'a formula (set of formulas) is satisfied by the matrix M' is un-
} Manuscrito - Rev. Int. Fil., Campinas, v. 34, n. 1, p. 365-388, jan.-jun. 2011. 


\begin{tabular}{l||l||l|llllll}
\hline$p$ & $\neg p$ & $p \vee q$ & 1 & 2 & 3 & 4 & 5 & 6 \\
\hline 1 & 6 & 1 & 1 & 1 & 1 & 1 & 1 & 1 \\
2 & 5 & 2 & 1 & 2 & 2 & 2 & 1 & 2 \\
3 & 4 & 3 & 1 & 2 & 3 & 1 & 3 & 3 \\
4 & 3 & 4 & 1 & 2 & 1 & 4 & 4 & 5 \\
5 & 2 & 5 & 1 & 1 & 3 & 4 & 5 & 5 \\
6 & 1 & 6 & 1 & 2 & 3 & 5 & 5 & 6 \\
\hline
\end{tabular}

\begin{tabular}{|c|c|c|c|c|c|c|c|c|c|c|c|c|c|c|}
\hline$p \wedge q$ & 1 & 2 & 3 & 4 & 5 & 6 & $p \rightarrow q$ & 1 & 2 & 3 & 4 & 5 & 6 & $L p$ \\
\hline 1 & 1 & 2 & 3 & 4 & 5 & 6 & 1 & 1 & 2 & 3 & 5 & 5 & 6 & 1 \\
\hline 2 & 2 & 2 & 3 & 4 & 6 & 6 & 2 & 1 & 1 & 3 & 5 & 5 & 5 & 3 \\
\hline 3 & 3 & 3 & 3 & 6 & 5 & 6 & 3 & 1 & 2 & 1 & 4 & 5 & 5 & 6 \\
\hline 4 & 4 & 4 & 6 & 4 & 5 & 6 & 4 & 1 & 2 & 3 & 1 & 3 & 3 & 6 \\
\hline 5 & 5 & 6 & 5 & 5 & 5 & 6 & 5 & 1 & 2 & 2 & 2 & 1 & 2 & 6 \\
\hline 6 & 6 & 6 & 6 & 6 & 6 & 6 & 6 & 1 & 1 & 1 & 1 & 1 & 1 & 6 \\
\hline
\end{tabular}

$$
p \leftrightarrow q=d f[(p \rightarrow q) \wedge(q \rightarrow p)]
$$

RMQ obeys the usual interdefinability between necessity $(L)$ and possibility $(M): M p=_{d f} \neg L \neg p$. Hence the matrices for all the seven positive modalities in RMQ are as follows:

\begin{tabular}{ccccccccc}
\hline$p$ & $\neg p$ & $L L p$ & $L p$ & $M L p$ & $p$ & $L M p$ & $M p$ & $M M p$ \\
\hline 1 & 6 & 1 & 1 & 1 & 1 & 1 & 1 & 1 \\
2 & 5 & 6 & 3 & 1 & 2 & 1 & 1 & 1 \\
3 & 4 & 6 & 6 & 6 & 3 & 1 & 1 & 1 \\
4 & 3 & 6 & 6 & 6 & 4 & 1 & 1 & 1 \\
5 & 2 & 6 & 6 & 6 & 5 & 6 & 4 & 1 \\
6 & 1 & 6 & 6 & 6 & 6 & 6 & 6 & 6 \\
\hline
\end{tabular}

As it follows from the definition, every well-formed formula (wff) of RMQ is unambiguously determined by a particular matrix, according derstood in Tarski's sense. Compare Tarski (1956, p. 41). 
to the definition in section 3.1, possessing either 6 or 36 or 216 etc. (in general $6^{n}, n=1,2, \ldots$ ) values. And any such particular matrix represents some well-formed formula (wff) of the system RMQ. ${ }^{5}$

The proofs for the properties of RMQ stated in the introduction (especially for properties (3), (4) and (9)) are given in Weingartner (2009, Chapter 3).

\subsection{Theorems of CPC which are only materially valid (but strictly invalid) in RMQ}

(1) $\neg p \rightarrow(p \rightarrow q)$

(2) $\neg p \rightarrow[p \rightarrow(q \wedge \neg q)]$

(3) $(p \rightarrow \neg p) \rightarrow[p \rightarrow(q \wedge \neg q)]$

(4) $p \rightarrow(p \vee q)$

(5) $p \rightarrow[p \vee(q \wedge \neg q)]$

(6) $p \rightarrow[(p \wedge q) \vee(p \wedge \neg q)]$

(7) $(p \rightarrow q) \rightarrow[(p \wedge r) \rightarrow(q \wedge r)]$

(8) $(p \rightarrow q) \rightarrow[(p \vee r) \rightarrow(q \vee r)]$

(9) $p \rightarrow(q \rightarrow p)$

(10) $p \rightarrow[(q \vee \neg q) \rightarrow p]$

(11) $p \rightarrow[q \rightarrow(p \wedge q)]$

(12) $(p \wedge q) \rightarrow(p \leftrightarrow q)$

(13) $(p \wedge q) \rightarrow(p \rightarrow q)$
Ex falso quodlibet

Ex falso quodlibet

Ex falso quodlibet

Redundant element(s)

Redundant element(s)

Redundant element(s)

Redundant element(s)

Redundant element(s)

Adding premise

Adding premise

Adding premise

Conjunction and implication

Conjunction and implication

\footnotetext{
${ }^{5}$ A similar system which differs slightly w.r.t. matrices of $\vee$ and $\rightarrow$, but agrees on $\neg, \wedge$ and modalities has been published as Weingartner (1968). This modal logic contains all theorems of CL in such a way that they hold necessarily (i.e., with $c v=2$ ). Compare Weingartner (2009, Section 6).
} 
(14) $(p \wedge q) \rightarrow[(q \wedge \neg q) \vee(q \wedge p)]$

(15) $(p \wedge q) \rightarrow[(p \wedge r) \vee(q \wedge \neg r)]$

(16) $[p \wedge(q \vee r)] \leftrightarrow[(p \wedge q) \vee(p \wedge r)]$

(17) $[(p \vee q) \wedge(p \vee r)] \leftrightarrow[p \vee(q \wedge r)]$

(18) $p \rightarrow[p \vee(p \wedge q)]$

(19) $p \rightarrow[p \wedge(p \vee q)]$

(20) $p \leftrightarrow[(p \wedge q) \vee(p \wedge \neg q)]$

(21) $p \vee(p \rightarrow q)$

(22) $(p \rightarrow q) \vee(p \rightarrow \neg q)$

(23) $(p \rightarrow q) \vee(\neg p \rightarrow q)$
Conjunction and disjunction Conjunction and disjunction

Distribution

Distribution

Absorption

Absorption

Irrelevant disjuncts Intuitionistically invalid $c v=3$ Intuitionistically invalid $c v=3$ Intuitionistically invalid $c v=3$

With the exception of the last three (21)-(23), the principles (CPC theorems) (1)-(11), (13)-(15), and (18)-(20) are separated as classically valid but replacement invariant: it is easily seen that at least one occurrence of the variable $q$ (or $p$ or $r$ ) in the consequent can be replaced by any arbitrary variable salva validitate (veritate) of the CPC theorem. The equivalence $p \leftrightarrow q$ in CPC theorem (12) has to be split into the two implications $(p \rightarrow q \wedge q \rightarrow p$ ) in order to see that. The direction $\rightarrow$ of (16) and (17) is forbidden by the restricted distribution $\mathrm{RD}$. The last three (21)-(23) cannot be designated that way because the main connective is not an implication, but a disjunction. However, for the system RMQ the form of the wff is not essential because the decision whether the wff is materially valid or strictly valid is determined by the cv of the matrix which represents the wff.

\subsection{Basic theorems of CPC which are strictly (or necessarily) valid (valid with $\mathrm{L}$ in front) in RMQ}

The main theorems which are strictly valid, are recognisable by their main connective $\Rightarrow$. For comparison also some principles which 
are strictly invalid (i.e., only materially valid, with $\rightarrow$ ) in RMQ, are listed below. All the main theorems which hold strictly (with $\Rightarrow$ ), are valid in a logic suitable for $\mathrm{QP}$.

(1) $(p \wedge q) \Leftrightarrow(q \wedge p)$

Commutation

(2) $(p \vee q) \Leftrightarrow(q \vee p)$

Commutation

(3) $[p \wedge(q \wedge r)] \Leftrightarrow[(p \wedge q) \wedge r]$

Association

(4) $p \Rightarrow p$

(5) $p \Leftrightarrow \neg \neg p$

Double negation

(6) $p \wedge q \Rightarrow p$

Simplification

(7) $p \wedge q \Rightarrow q$

Simplification

(8) $p \vee p \Rightarrow p$

Simplification

(9) $[(p \rightarrow q) \wedge p] \Rightarrow q$

Modus ponens

(10) $[(p \rightarrow q) \wedge \neg q] \Rightarrow \neg p$

Modus tollens

(11) $(p \rightarrow q) \Rightarrow(\neg q \rightarrow \neg p)$

Contraposition

(12) $[(p \rightarrow q) \wedge(q \rightarrow r)] \Rightarrow(p \rightarrow r)$

Hypothetical syllogism

(transitivity of $\rightarrow$ )

(13) $[(p \vee q) \wedge \neg p] \Rightarrow q$

Disjunctive syllogism

(14) $(p \wedge q) \Rightarrow \neg(\neg p \vee \neg q)$

De Morgan's law

(15) $(p \vee q) \Rightarrow \neg(\neg p \wedge \neg q)$

De Morgan's law

(16) $(\neg p \wedge \neg q) \Rightarrow \neg(p \vee q)$

De Morgan's law

(17) $(\neg p \vee \neg q) \Rightarrow \neg(p \wedge q)$

De Morgan's law

(18) $\neg(\neg p \vee \neg q) \rightarrow(p \wedge q)$

De Morgan's law

(19) $\neg(\neg p \wedge \neg q) \rightarrow(p \vee q)$

De Morgan's law

Manuscrito - Rev. Int. Fil., Campinas, v. 34, n. 1, p. 365-388, jan.-jun. 2011. 
(20) $\neg(p \vee q) \rightarrow(\neg p \wedge \neg q)$

De Morgan's law

(21) $\neg(p \wedge q) \rightarrow(\neg p \vee \neg q)$

De Morgan's law

Looking at De Morgan's laws (14)-(21) shows that strict validity in RMQ interprets a negation before parenthesis of a compound wff as weaker than the negation which is applied immediately to the wff. This coincides partially with intuitionistic logic, such that Principles (18)-(21) hold only materially in RMQ.

(22) $[(p \wedge q) \vee(p \wedge r)] \Rightarrow[p \wedge(q \vee r)]$

Distribution

$(23)[p \wedge(q \vee r)] \rightarrow[(p \wedge q) \vee(p \wedge r)]$

Distribution

(24) $[p \vee(q \wedge r)] \Rightarrow[(p \vee q) \wedge(p \vee r)]$

Distribution

$(25)[(p \vee q) \wedge(p \vee r)] \rightarrow[p \vee(q \wedge r)]$

Distribution

(26) $[(p \wedge q) \vee(p \wedge \neg q)] \Rightarrow[p \wedge(q \vee \neg q)]$ Instance of distribution

(27) $[(p \wedge q) \vee(p \wedge \neg q)] \Rightarrow p$

(28) $[p \rightarrow(q \wedge r)] \Rightarrow[(p \rightarrow q) \wedge(p \rightarrow r)]$

(29) $[(p \rightarrow q) \wedge(p \rightarrow r)] \rightarrow[p \rightarrow(q \wedge r)]$

(30) $[(p \rightarrow r) \vee(q \rightarrow r)] \Rightarrow[(p \wedge q) \rightarrow r]$

$(31)[(p \wedge q) \rightarrow r] \rightarrow[(p \rightarrow r) \vee(q \rightarrow r)]$

$(32)[r \rightarrow(p \rightarrow q)] \Rightarrow[(r \wedge p) \rightarrow q]$

$(33)[(r \wedge p) \rightarrow q] \rightarrow[r \rightarrow(p \rightarrow q)]$

(34) $\neg(p \wedge \neg p)$

Principle of noncontradiction strongly valid

(35) $p \vee \neg p$ Principle of Excluded Middle or tertium non datur strongly valid

Manuscrito - Rev. Int. Fil., Campinas, v. 34, n. 1, p. 365-388, jan.-jun. 2011. 
The strong validity $(c v=1)$ of the principle of noncontradiction means that no modal operator can change the value, since it is 1 . Also: no modal operator can change the value 6 of the contradiction (see the Table in Section 3.1). Since $\neg p$ is the mirror image of $p, p \wedge \neg p$ has the maximum value false, whereas $\neg(p \wedge \neg p)$ has the maximum value true. The reason for it is the matrix of negation (mirror image) and of conjunction, which has values 6 in the diagonal from right top to left bottom. Similarly, the strong validity of the tertium non datur is caused by the matrix of negation and of disjunction, which has values 1 in the same diagonal. Some of these basic assumptions concerning the matrices will now be changed in order to receive a system which is paraconsistent in a weak or stronger sense.

\section{MATRIX BASED PARACONSISTENT LOGICS}

In this chapter we present two paraconsistent logics. A weak paraconsistent logic which satisfies conditions DC2 and DC3 of Newton da Costa. A system which satisfies DC2 was called paraconsistent by Priest (Priest (2000, p. 224)). It seems to me that also DC3 should be accepted as a necessary condition, otherwise DC2 alone seems to be leading to "anything goes". The second system is stronger as it satisfies all three criteria DC1, DC2 and DC3 of Newton da Costa.

Since both systems are finite matrix-systems they are easily proved to be decidable and consistent themselfes. Moreover both systems satisfy DC3 of Newton da Costa in the sense that all valid theorems of CPC are classically or materially valid $(c v=3)$ but those of them which do not make too strong classical assumptions are also strictly valid (for a detailed discussion of "too strong classical assumptions" see Weingartner (2010, Chapter 2)). By this property which is a maincharacteristic of RMQ they more or less separate theorems which make too strong classical assumptions (and when applied lead to difficul- 
ties) - as being only materially valid but strictly invalid - from those which do not lead to difficulties in the application - as being strictly valid.

\subsection{The paraconsistent system RMQP}

RMQP results from RMQ by weakening its negation. In RMQ the negation of $p, \neg p$, has the matrix 654321 if $p$ has the matrix 123456 . The negation of RMQP $(\sim)$ results from the negation of RMQ just by replacing the truth-value 3 by a truth-value 2 . Thus if $p$ has the matrix 123456 then $\sim p$ has the matrix 654221 . All other matrices of disjunction, conjunction and implication are the same as in RMQ. The resulting system is however already quite different from RMQ, although there is only a slight difference in the matrix of negation.

Concerning the theorems of RMQP (see sections 4.1.1 and 4.1.2) it is easily understandable that all theorems of RMQ which do not contain a negation sign are the same theorems in RMQP. From this it follows immediately that some virtues of RMQ are directly transmitted to RMQP (section 4.1.2). For example the traditionally and presently important theorems of modus ponens (10), commutation (1),(2), simplification (7)-(9), hypothetical syllogism (= transitivity of implication) (12) are all strictly valid $(c v=2)$. Moreover exactly those distribution laws (15),(17) which do not lead to difficulties in the application to Quantum Physics (from disjuncts to conjuncts) are strictly valid, whereas those which make too strong classical assumptions (from conjuncts to disjuncts (16),(18)) are strictly invalid (only classically or materially valid). Of the eight classically valid De Morgan Laws only two (13),(14) are strictly valid in RMQP; these are exactly the ones which are also intuitionistically valid.

On the other hand many theorems of CPC which lead to paradoxes and difficulties in different domains (see section $\mathbf{2 . 1}$ above) are strictly 
invalid in both RMQ and RMQP, also if the two different types of negation are included (section 4.1.1). Examples are: Ex falso quodlibet (1)-(3), adding premise (9)-(11), principle of addition (4), absorption (18),(19), conjunction implies implication (13), general commensurability (20), Bell's inequalities (15) ...etc. Moreover in RMQP the principle ex contradictione quodlibet ("principle of explosion") (26), disjunctive syllogism (25), contraposition (21) and double negation (27)-(29) are strictly invalid (only classically or materially valid).

\subsubsection{Theorems of CPC which are strictly invalid (but only classically or materially valid, $c v=3$ ) in RMQP}

(1) $\sim p \rightarrow(p \rightarrow q)$

(2) $\sim p \rightarrow[p \rightarrow(q \wedge \sim q)]$

(3) $(p \rightarrow \sim p) \rightarrow[p \rightarrow(q \wedge \sim q)]$

(4) $p \rightarrow(p \vee q)$

(5) $p \rightarrow[p \vee(q \wedge \sim q)]$

(6) $p \rightarrow[(p \wedge q) \vee(p \wedge \sim q)]$

(7) $(p \rightarrow q) \rightarrow[(p \wedge r) \rightarrow(q \wedge r)]$

(8) $(p \rightarrow q) \rightarrow[(p \vee r) \rightarrow(q \vee r)]$

(9) $p \rightarrow(q \rightarrow p)$

(10) $p \rightarrow[(q \vee \sim q) \rightarrow p]$

(11) $p \rightarrow[q \rightarrow(p \wedge q)]$

(12) $(p \wedge q) \rightarrow(p \leftrightarrow q)$

(13) $(p \wedge q) \rightarrow(p \rightarrow q)$

(14) $(p \wedge q) \rightarrow[(q \wedge \sim q) \vee(q \wedge p)]$
Ex falso quodlibet

Ex falso quodlibet

Ex falso quodlibet

Redundant element(s)

Redundant element(s)

Redundant element(s)

Redundant element(s)

Redundant element(s)

Adding premise

Adding premise

Adding premise

Conjunction and implication

Conjunction and implication

Conjunction and disjunction 
(15) $(p \wedge q) \rightarrow[(p \wedge r) \vee(q \wedge \sim r)]$

(16) $[p \wedge(q \vee r)] \leftrightarrow[(p \wedge q) \vee(p \wedge r)]$

(17) $[(p \vee q) \wedge(p \vee r)] \leftrightarrow[p \vee(q \wedge r)]$

(18) $p \rightarrow[p \vee(p \wedge q)]$

(19) $p \rightarrow[p \wedge(p \vee q)]$

(20) $p \leftrightarrow[(p \wedge q) \vee(p \wedge \sim q)]$

$(21)(p \rightarrow q) \rightarrow(\sim q \rightarrow \sim p)$

(22) $p \vee(p \rightarrow q)$

(23) $(p \rightarrow q) \vee(p \rightarrow \sim q)$

(24) $(p \rightarrow q) \vee(\sim p \rightarrow q)$

(25) $[(p \vee q) \wedge \sim p] \rightarrow q$

(26) $(p \wedge \sim p) \rightarrow q$

(27) $p \rightarrow \sim \sim p$

(28) $\sim \sim p \rightarrow p$

(29) $\sim p \rightarrow \sim \sim \sim p$
Conjunction and disjunction

Distribution Distribution Absorption Absorption Irrelevant disjuncts Contraposition Intuitionistically invalid $c v=3$ Intuitionistically invalid $c v=3$ Intuitionistically invalid $c v=3$ Disjunctive syllogism ex contradictione quodlibet Double Negation Double Negation intuitionistically valid

\subsubsection{Theorems of CPC which are strictly valid in RMQP} $(\Rightarrow)$ (including some strictly invalid theorems for comparison $(\rightarrow))$

(1) $(p \wedge q) \Leftrightarrow(q \wedge p)$

Commutation

(2) $(p \vee q) \Leftrightarrow(q \vee p)$

Commutation

(3) $[p \wedge(q \wedge r)] \Leftrightarrow[(p \wedge q) \wedge r]$

Association

(4) $p \Rightarrow p$

(5) $\sim \sim p \Rightarrow \sim p$

Manuscrito - Rev. Int. Fil., Campinas, v. 34, n. 1, p. 365-388, jan.-jun. 2011. 
(6) $\sim \sim p \Leftrightarrow \sim \sim \sim \sim p$

(7) $p \wedge q \Rightarrow p$

Simplification

(8) $p \wedge q \Rightarrow q$

Simplification

(9) $p \vee p \Rightarrow p$

Simplification

(10) $[(p \rightarrow q) \wedge p] \Rightarrow q$

Modus ponens

(11) $[(p \rightarrow q) \wedge \sim q] \Rightarrow \sim p$

Modus tollens

(12) $[(p \rightarrow q) \wedge(q \rightarrow r)] \Rightarrow(p \rightarrow r) \quad$ Hypothesis syllogism (transitivity of $\rightarrow$ )

(13) $(\sim p \vee \sim q) \Rightarrow \sim(p \wedge q)$ De Morgan's law (intuitionistically valid)

(14) $\sim(p \vee q) \Rightarrow(\sim p \wedge \sim q)$ De Morgan's law (intuitionistically valid)

(15) $[(p \wedge q) \vee(p \wedge r)] \Rightarrow[p \wedge(q \vee r)]$

Distribution

(16) $[p \wedge(q \vee r)] \rightarrow[(p \wedge q) \vee(p \wedge r)]$

Distribution

(17) $[p \vee(q \wedge r)] \Rightarrow[(p \vee q) \wedge(p \vee r)]$

Distribution

(18) $[(p \vee q) \wedge(p \vee r)] \rightarrow[p \vee(q \wedge r)]$

Distribution

(19) $[(p \wedge q) \vee(p \wedge \sim q)] \Rightarrow[p \wedge(q \vee \sim q)]$ Instance of distribution

(20) $[(p \wedge q) \vee(p \wedge \sim q)] \Rightarrow p$

(21) $[p \rightarrow(q \wedge r)] \Rightarrow[(p \rightarrow q) \wedge(p \rightarrow r)]$

$(22)[(p \rightarrow q) \wedge(p \rightarrow r)] \rightarrow[p \rightarrow(q \wedge r)]$

(23) $[(p \rightarrow r) \vee(q \rightarrow r)] \Rightarrow[(p \wedge q) \rightarrow r]$

$(24)[(p \wedge q) \rightarrow r] \rightarrow[(p \rightarrow r) \vee(q \rightarrow r)]$

$(25)[r \rightarrow(p \rightarrow q)] \Rightarrow[(r \wedge p) \rightarrow q]$

(26) $[(r \wedge p) \rightarrow q] \rightarrow[r \rightarrow(p \rightarrow q)]$ 
$(27) \sim(p \wedge \sim p)$

$c v=2$ Principle of non-contradiction

(28) $p \vee \sim p \quad c v=2$ Principle excluded middle (tertium non datur)

\subsubsection{Desiderata satisfied by RMQP}

The following desiderata are satisfied by RMQP:

(1) RMQP is consistent and decidable.

(2) DC3 of Newton da Costa. RMQP includes CPC materially (but not strictly).

(3) DC2 of Newton da Costa. The principle ex contradictione quodlibet is strictly invalid.

(4) Important principles for any kind of logic (also traditionally important) are strictly valid in RMQP (cf. 4.1 and 4.1.2 above).

(5) Theorems of CPC which lead to difficulties when applied to empirical sciences (violating the replacement criterion $\mathrm{RC}$ ) are stricly invalid in RMQP (cf. 4.1 and 4.1.1 above).

(6) Theorems of CPC which make specific classical assumptions and which cause difficulties in the application to Quantum Physics are strictly invalid in RMQP:

(a) CPC-theorems which claim universal commensurability

(b) CPC-theorems which claim both directions of distributivity

(c) CPC-theorems which claim the propositional versions of Bell's inequalities

(7) Despite the advantages of (1)-(6) the principle tertium non datur $p \vee \sim p$, and the principle of non-contradiction, $\sim(p \wedge \sim p)$, are strictly valid in RMQP. 


\subsubsection{Comparison with RMQ}

In general there are many positive similarities between RMQ and RMQP which is shown especially by the points (4)-(7) of $\mathbf{4 . 1 . 3}$ above.

Disadvantages of RMQP w.r.t. RMQ are that the principles of Double Negation, Contraposition and several of the De Morgan Laws are strictly invalid. That Double Negation is not strictly valid coincides with Da Costa's $C_{1}^{=}$calculus of 1974 . Also in $C_{1}^{=}$the principle of contraposition needs the additional premise of $\sim(q \wedge \sim q)$ although the latter is strictly valid in RMQP. That Disjunctive Syllogism is strictly invalid may seem an advantage to some and a disadvantage to others. According to our results from investigating paradoxes in different domains including those concerning the application in Quantum Physics (cf Weingartner, Schurz (1986), Weingartner (2001)), Disjunctive Syllogism is harmless. On the other hand it is manifest that the principle of addition is the culprit for many paradoxes (although the usual Relevant Logicians defend it against Disjunctive Syllogism).

As an advantage w.r.t. RMQ one may see that condition DC2 of Da Costa is satisfied in RMQP.

\subsubsection{Axiomatisability}

It may be asked how RMQ is axiomatisable; that is, how the properties of the above matrices can be formulated with the help of axioms. Such a task was solved for the matrix system mentioned in Note 5 above by Czermak (1981) which can be represented by a set of 33 axioms. They may be reduced by independence proofs (and choosing a different basis for propositional logic) to 26. Since RMQ is more complicated than this system (since it distinguishes materially valid theorems of CPC from strictly valid ones) it is probable that RMQ needs more than 33 (26) axioms to represent its matrices. The same will be true for the system RMQP. This only shows that the matrix-based logic RMQ and also its paraconsistent alternative RMQP are very specific. 


\subsection{The paraconsistent system RMQP*}

$\mathrm{RMQP}^{*}$ results from RMQ by weakening its conjunction. For values 3 and 4 it holds in RMQ that $3 \wedge 4=6$ and $4 \wedge 3=6$. In RMQP* it holds that $3 \wedge 4=4$ and $4 \wedge 3=4$. All other matrices of negation, disjunction, implication and the remaining part in the matrix of conjunction are the same as in RMQ. Despite the small difference the resulting system is quite different from RMQ; in this case much more different from RMQ than RMQP.

Like RMQP, RMQP* is consistent and decidable. RMQP* satisfies all three criteria DC1, DC2 and DC3 of Newton da Costa. However, generally speaking, $\mathrm{RMQP}^{*}$ destroys one basic idea of RMQ. This idea was to separate those $\mathrm{CPC}$-theorems which make too strong classical assumptions and lead to paradoxes and difficulties in the application to different domains from those which do not cause difficulties and have been important logical principles in the tradition up to the present time. The former are strictly invalid in RMQ (only materially valid) and the latter are strictly valid in RMQ. RMQP behaves in a similar way w.r.t. this basic idea realised in RMQ.

On the other hand in $\mathrm{RMQP}^{*}$ the strict validity of most principles of RMQ and RMQP is reduced to classical or material validity such that no reasonable difference remains. This can be seen from the following results on theorems in RMQP*:

(1) RMQP* is consistent and decidable.

(2) RMQP* satisfies all three criteria DC1, DC2 and DC3 of Newton da Costa.

(3) All CPC-theorems which do not contain any conjunction behave like in RMQ. These are in section 3.2 above: (1), (4), (9), (10), (21), (22), (23); moreover (2), (3) und (15) are also strictly invalid.

In section 3.3 above: (2), (4), (5), (8), (11), (35). Moreover (1) 
because of the symmetry of conjunction.

This means that Double Negation, Contraposition and tertium non datur are strictly valid in RMQP*.

(4) Many important principles for any logic are however strictly invalid (only classically or materially valid) in RMQP* . For example: modus ponens, modus tollens, simplification, hypothetical syllogism (= transitivity of implication), all 8 De Morgan Laws, all laws of distribution.

This means that RMQP* puts into one pot both, the above important principles and those principles violating criteria $\mathrm{RC}$ and $\mathrm{RD}$ and leading to difficulties and paradoxes when applied to empirical sciences.

(5) Though RMQP* throws out from the strictly valid theorems those which claim universal commensurability and (the propositional version of) Bell's inequalities, it also throws out all (directions of the) distribution laws.

It remains the task of further investigation whether one could find such an alternative of RMQ which on the one hand satisfies all three criteria of Newton da Costa and on the other makes a reasonable separation of strictly valid theorems on the one hand and materially valid theorems on the other in the sense of RMQ and RMQP. From our investigations so far it seems more reasonable to keep the principle of non-contradiction strictly valid for any basic logic suitably applicable to empirical sciences. That means that satisfying criteria DC2 and DC3 of Newton da Costa seems to be a better solution. 


\section{REFERENCES}

CZERMAK, J. "Eine endliche Axiomatisierung von SS1M". In Morscher et al., editors. Philosophie als Wissenschaft. Essays in Scientific Philosophy. Bad Reichenhall: Comes. p. 245-258, 1981.

DA COSTA, N. "On the Theory of Inconsistent Formal Systems". Notre Dame Journal of Formal Logic, 15, p. 497-510, 1974.

MITTELSTAEDT, P. Quantum Logic. Dordrecht, The Netherlands: Reidel, 1978.

- "Does quantum physics require a new logic?" In Weingartner, P., editor. Alternative Logics. Do Sciences Need Them? Heidelberg, Germany: Springer. p. 269-284, 2004.

PRIEST, G. "Motivation of paraconsistency: The slippery slope from classical logic to dialethism." In Batens, D., Mortensen, Ch., Priest, G., and van Bendegem, J. P., editors. Frontiers in Paraconsistent Logics. London: Research Studies Press, p. 223-233, 2000 .

SCHURZ, G. "Relevant deduction". Erkenntnis, 35, p. 391-437, 1991.

SCHURZ, G., WEINGARTNER, P. "Verisimilitude defined by relevant consequence elements. A new reconstruction of Popper's original idea". In Kupiers, T., editor. What is Closer-to-the-Truth? Amsterdam, The Netherland: Rodopi, p. 47-77, 1987.

— . "Zwart and Franssen's impossibility theorem holds for possible-world-accounts but not for consequence-accounts to versimilitude". Synthese, 2009.

TARSKI, A. Logic Semantics and Metamathematics. Oxford, UK: Oxford University Press, 1956. 
WEINGARTNER, P. "Modal logics with two kinds of necessity and possibility". Notre Dame Journal of Formal Logic, 9(2), p. 97$159,1968$.

. "Reasons for filtering classical logic". In Batens, D., Mortensen, Ch., Priest, G., and van Bendegem, J. P., editors. Frontiers in Paraconsistent Logics. London: Research Studies Press, p. $315-327,2000$.

. "Applications of logic outside logic and mathematics: Do such applications force us to deviate from classical logic?!" In Stelzner, W., editor. Zwischen traditioneller und moderner Logik. Paderborn: Mentis, p. 53-64, 2001.

. "Reasons from science for limiting classical logic". In Weingartner, P., editor. Alternative Logics. Do Sciences Need Them? Heidelberg, Germany: Springer. p. 233-248, 2004.

_ . "Matrix-Based Logic for Application in Physics". The Review of Symbolic Logic, 2(1), p. 132-163, 2009.

_. "Basis Logic for Application in Physics and its Intuitionistic Alternative." In Busch, P., editor. Essays in Honour of Peter Mittelstaedt. Foundations of Physics, v. 40, p. 1578-1596, 2010.

WEINGARTNER, P., SCHURZ, G. "Paradoxes solved by simple relevance criteria." Logique et Analyse, 113, p.3-40, 1986. 